\title{
La parenthèse en suspens notes sur la traduction Italienne des Géorgiques
}

\section{Emilia Surmonte}

\section{(2) OpenEdition \\ 1 Journals}

Édition électronique

URL : http://journals.openedition.org/ccs/962

DOI : $10.4000 /$ ccs.962

ISSN : 2558-782X

Éditeur :

Presses universitaires de Rennes, Association des lecteurs de Claude Simon

\section{Édition imprimée}

Date de publication : 30 avril 2015

Pagination : 169-178

ISBN : 9782753539990

ISSN : 1774-9425

\section{Référence électronique}

Emilia Surmonte, "La parenthèse en suspens notes sur la traduction Italienne des Géorgiques »

Cahiers Claude Simon [En ligne], 10 | 2015, mis en ligne le 22 septembre 2017, consulté le 19 avril 2019.

URL : http://journals.openedition.org/ccs/962 ; DOI : 10.4000/ccs.962 


\title{
LA PARENTHÈSE EN SUSPENS NOTES SUR LA TRADUCTION ITALIENNE DES GÉORGIQUES
}

\author{
Emilia SURMONTE \\ Università degli Studi di Napoli « L'Orientale »
}

\section{TRADUCTIONS ET TRADUCTEURS}

L'œuvre de Claude Simon s'impose rapidement à la critique et au public italiens au début des années 60 , comme en témoignent les dates des premières traductions. L'Herbe ${ }^{1}$ sort en France en 1958 et trois ans plus tard chez Einaudi. L'année suivante, en 1962, la même maison d'édition publie la version italienne de La Route des Flandres ${ }^{2}$ (traduite par Guido Neri), parue en France seulement deux ans plus tôt. Suivra en 1965 Le Palace $^{3}$ (traduit par Gioia Zannino Angiolillo), trois ans après sa publication aux Éditions de Minuit.

Compte tenu de la complexité de l'écriture simonienne, ces données montrent que la critique italienne était attentive à l'œuvre de Claude Simon et que la machine éditoriale de l'époque avait dû se mettre en marche dès la parution de ses œuvres en France. On doit à Bruno Fonzi d'abord, conseiller éditorial d'Einaudi, et ensuite à Guido Neri, professeur de Littérature française à l'université de Bologne et également conseiller éditorial d'Einaudi, les premières traductions des ouvres de Claude Simon en Italie. Comme le rappelle Anna Bucarelli dans son article de 2011, "La réception de l'œuvre simonienne en Italie », c'est Guido Neri qui «a joué un rôle essentiel dans

1. C. Simon, L'erba, trad. di Bruno Fonzi, Torino, Einaudi, 1961.

2. C. Simon, La strada delle Fiandre, trad. di Guido Neri, Torino, Einaudi, 1962.

3. C. Simon, Il palace, trad. di Gioia Zannino Angiolillo, Torino, Einaudi, 1965. 
l'introduction en Italie de nombreux auteurs français [...]. On lui doit des traductions remarquables de La Route des Flandres, d'Histoire ${ }^{4}$ et de La Chevelure de Bérénice ${ }^{5}$.

Claude Simon continue d'être traduit en italien dans la première moitié des années 70 (Histoire sort en 1971 et Tryptique ${ }^{7}$, traduit par Nanni Balestrini, en 1975 ) et d'être publié par Einaudi. Une période de silence commence juste après, à quelques rares exceptions près ${ }^{8}$, et ne reprendra qu'après l'attribution du Prix Nobel en 1985, où l'on assiste à une floraison d'études critiques et d'articles qui a continué pendant les années 90 et s'est intensifiée juste après la mort de l'auteur en 2005, comme le montre Anna Bucarelli ${ }^{9}$.

Un destin quelque peu différent est réservé aux traductions. Reprises après 1985, elles ont connu une longue halte de 1999 (Leçon de choses $^{10}$, traduite par Roberto Marro) à 2012.

En 1975, Einaudi lance un projet de traduction de La Bataille de Pharsale ${ }^{11}$ qui reste sans suite jusqu'en 1987. En annonçant dans la presse la publication de cette œuvre, traduite par Dianella Silvatico Estense, Giovanni Bogliolo, professeur de Littérature française à l'université d'Urbino et futur traducteur de $L^{\prime}$ Acacia ${ }^{12}$ en 1994, analyse les causes de ce silence éditorial et défend avec vigueur, contre tout préjugé, l'originalité de l'écriture simonienne et sa lisibilité:

Si le bruit engendré par le Nobel n'a pas suffi à rendre l'écrivain populaire, il aura au moins servi à détruire sa réputation d'inaccessibilité, à divulguer le sens de sa recherche et à susciter l'intérêt pour cet expérimentateur solitaire et son travail de la part de ceux qui s'étaient contentés de formules (le «nouveau roman » notamment) qui peuvent caractériser tout au plus un moment, court, lointain et dépassé, de ses évolutions ${ }^{13}$.

4. C. Simon, Storia, trad. di Guido Neri, Torino, Einaudi, 1971.

5. C. Simon, La chioma di Berenice, trad. di Guido Neri, edizione bilingue, Padova, Liviana, 1986.

6. Voir A. BUCARELLI, "La réception de l'œuvre simonienne en Italie", Cahiers Claude Simon, n. 7, Anne-Yvonne Julien (dir.), Perpignan, Presses universitaires de Perpignan, 2011, p. 183. L'étude d'Anna Bucarelli est à l'heure actuelle une référence incontournable pour toute documentation sur la bibliographie italienne associée à Claude Simon et à la traduction de ses œuvres.

7. C. Simon, Trittico, trad. di Nanni Balestrini, Torino, Einaudi, 1975.

8. "C'est à partir des années 1980, en effet que la critique italienne consacre à Claude Simon des études de plus en plus nombreuses, témoignant de l'intérêt grandissant qui est porté à son œuvre ». A. Bucarelli, "La réception de l'œuvre simonienne en Italie ", Cahiers Claude Simon, n. 7, op.cit., p. 195.

9. Ibid.

10. C. Simon, Il senso delle cose, trad. di Roberto Marro, Torino, Testo \& Immagine, 1999.

11. C. Simon, La battaglia di Farsalo, trad. di Dianella Selvatico Estense, Torino, Einaudi, 1987.

12. C. Simon, L'acacia, trad. di Giovanni Bogliolo, Torino, Einaudi, 1994.

13. Giovanni Bogliolo, «Simon a Farsalo scompone il tempo in una sinfonia », Tuttolibri, 21 février 1987, p. 2 : «Il clamore del Nobel se non è bastato a dare la popolarità allo scrittore, è servito almeno a sfatare la fama 
Dans le même article, Bogliolo réfléchit sur la relation scripturale qui lie cette œuvre aux Géorgiques: "On aperçoit déjà l'esquisse de l'extraordinaire symphonie narrative des Géorgiques, qu'aucune annonce éditoriale ne promet à l'heure actuelle mais que les vingt-cinq lecteurs de Simon ont le droit (et la patience) d'attendre ${ }^{14}$. ".

Ce n'est qu'en 2012 que le vœu de Giovanni Bogliolo se concrétise, grâce à la maison d'édition Lavieri ${ }^{15}$, 13 ans après la publication de Leçon de choses. Complétée par deux postfaces critiques - l'une "Tradurre Simon » sur la traduction, l'autre "Claude Simon. Dal plurale al plurale " sur l'œuvre ${ }^{16}-$, la publication en italien des Géorgiques, que j'ai traduites, comble un vide salué avec faveur dans la presse générale et spécialisée ${ }^{17}$.

Deux autres traductions sont annoncées sur le site de Nonostante Edizioni dans la collection Scrittura Bianca. La première, publiée en octobre 2014, est une réédition de L'Herbe ${ }^{18}$ traduite par Bruno Fonzi, accompagnée cette fois d'une postface d'Alastair B. Duncan qui ne figurait pas dans la première édition Einaudi. La deuxième est Le Tramway ${ }^{19}$, œuvre inédite en Italie, traduite par Stefania Ricciardi, avec une postface de Patrick Longuet, encore en préparation à cette date.

Einaudi domine la scène éditoriale jusqu'aux années 70 et continue de publier des ouvres de Claude Simon tout au long des années 90 (La Bataille de Pharsale, déjà citée, le Discours de Stockholm, dans la version de Sergio Atzeni ${ }^{20}$

della sua inaccessibilità, a divulgare il senso della sua ricerca e a destare attorno al suo lavoro l'interesse di quanti per questo solitario sperimentatore si erano accontentati di formule (il "nouveau roman" appunto) che al massimo possono caratterizzare un momento, breve, lontano, superato, delle sue evoluzioni ».

14. Ibid.: "Già si intravede l'abbozzo della straordinaria sinfonia narrativa delle Géorgiques, che nessun annuncio editoriale per ora promette ma che i venticinque lettori di Simon hanno il diritto (e la pazienza) di aspettare ".

15. C. Simon, Le Georgiche, trad. di Emilia Surmonte, collana Arno (dirigée par Domenico Pinto), S. Angelo in Formis, Lavieri, 2012.

16. Voir ibid. Postfaces «Tradurre Simon », p. 357-361 et «Claude Simon. Dal plurale al plurale » (p. 363-374).

17. Voir à ce propos les articles de Enzo di Mauro, "Navigare a vista nel mare del '900 ", Il fatto quotidiano, 24 août 2013, Stefano Gallerani, "Simon. Pluralità e montaggio per sconfessare l'ordine della Storia ", Alias, 29 septembre 2013; Marco Ferri, "L'Eneide di Alessandro Fo e Le Georgiche di Claude Simon ", Filobus66.it, 11 septembre 2013, Giancarlo Alfano, Claude Simon. Le Georgiche, dans «Il libro del mese ", Alfabeta 2, septembre 2013. Tous les articles sont consultables sur le site de Lavieri editore: http://www. lavieri.it/rassegna/rassegna_simon.php

18. C. Simon, L'erba, trad. di Bruno Fonzi, Trieste, Nonostante Edizioni, 2014.

19. C. Simon, Il tram, trad. di Stefania Ricciardi, Trieste, Nonostante Edizioni, en préparation.

20. Discours de Stockholm, trad. de Sergio Atzeni, dans AA.VV., Il giro del mondo in diciotto autori. La narrativa straniera Einaudi 1994 nelle pagine autobiografiche degli scrittori, Torino, Einaudi, 1994. 
et L'Acacia, traduit par Giovanni Bogliolo) même si d'autres éditeurs s'intéressent à cet écrivain après l'attribution du Nobel. Liviana publie La Chevelure de Bérénice (traduite par Guido Neri) en 1986, Utet réédite La Route des Flandres et Histoire (trad. Guido Neri, éd. Anna Maria Mossetto) en 1989 dans sa collection "I Nobel ", Tracce Discours de Stockholm (traduit par Anna Maria Salvatore) en 1992, L'Obliquo L'Invitation (trad. et éd. Marco Ferri) en 1993, Testo \& Immagine Leçon de choses (traduite par Roberto Marro) en 1999, Lavieri Les Géorgiques (traduites par Emilia Surmonte) en 2012, Nonostante Edizioni L'Herbe en 2014 et Le Tramway, publication in fieri.

La raréfaction de la présence de Einaudi n'est pas sans conséquence pour la diffusion de l'œuvre de Claude Simon en Italie, car les autres maisons d'éditions ont un pouvoir publicitaire et une pénétration sur le marché moindre, ce qui contribue à faire encore de Simon dans le Belpaese un écrivain à "vingt-cinq lecteurs ", bien que son projet d'écriture s'avère aujourd'hui beaucoup plus proche des modalités de lecture e-textuelle - dialogique et plurilogique -, que de celles de son époque. Car Simon construit ses textes comme des hypertextes qui, par les contraintes du support page/ligne, se présentent sous forme de succession linéaire, mais où les signes typographiques (parenthèses/ponctuation, romain, italique, pour ne citer que les plus récurrents) assument le rôle de liens (au sens informatique) vers d'autres textes, comme digressions internes, approfondissements, divagations, dans une alternance de processus de de-structuration, costructuration, re-structuration. Un système scriptural que David Zemmour définit ainsi :

En effet, un roman de Simon est à la fois texte et textes, saisi dans une esthétique de patchwork qui réactive l'étymologie du texte tissu, l'assemblage de fragments disparates et hétérogènes finissant par former un tout solidaire et indissociable. Le texte simonien est pris dans ce paradoxe, hétérogène sans être hétéroclite (ou hétérogène dans ses parties mais homogène dans le tout) ${ }^{21}$.

La variété des maisons d'éditions a entraîné aussi une variété de traducteurs. Compte tenu des travaux en cours, sur un total de quinze œuvres, il y a treize traducteurs différents. Exception faite pour Guido Neri, auteur de trois traductions, tous les autres se sont limités, jusqu’à présent, à une seule œuvre pour différentes raisons qu'il n'est pas utile d'évoquer ici.

Ce qui importe, dans ce contexte, est le fait que chacune de ces traductions porte les signes d'un dialogue important avec le texte de départ, donnant lieu à des approches et à des solutions originales, en faisant de chacune d'elle un uni-

21. D. Zemmour, Une syntaxe du sensible. Claude Simon et l'écriture de la perception, PUPS, 2008, p. 73-74. 
cum, ce qui constitue, certainement, une limite pour le lecteur italien, exclu, au moins en partie, du réseau intertextuel - fait de renvois factuels, thématiques, sémantico-syntaxiques et émotionnels - que Simon construit patiemment tout le long de son œuvre, comme une toile d'araignée, comme « monde parallèle, version mythique de ses propres origines, fragmentaire mais cohérent, et qui hante le souvenir ${ }^{22}$ ". Monde qui trouve finalement son centre dans Les Géorgiques, comme le relève Alastair Duncan ${ }^{23}$.

Mais cette caractéristique qui investit les traductions italiennes s'avère, tout compte fait, une ressource, car elle ouvre la voie à un enrichissement des perspectives exégétiques ainsi qu’à une réflexion sur les problématiques liées à la traduction des œuvres littéraires à l'époque contemporaine, et en particulier à celles des œuvres de Claude Simon.

\section{LA TRADUCTION DES GÉORGIQUES ET LA NARRATION DU JE}

Toute traduction est fille de son temps. Entre les précédentes et celle que j’ai réalisée, beaucoup de changements se sont produits, non seulement pour ce qui est des modalités de lecture et du rapport à la textualité, comme je le rappelais plus haut, mais aussi dans la manière de concevoir la traduction littéraire, plus franchement attentive, aujourd'hui, à une fidélité substantielle et formelle au texte de départ, et cela aussi en vertu de la formation spécialisée des traducteurs.

Les Géorgiques sont un exemple très original de narration temporelle de la subjectivité24, de ce roman du "je », comme l'appelle Philippe Forest ${ }^{25}$, où le vécu révèle entièrement sa nature et sa structure multiples, sa matière plurielle toujours inaccomplie et sans solution, fragmentaire et mouvante. Et cela à partir du titre, qui reprend celui des Georgica de Virgile mais aussi l'anthroponyme Georges ("travailleur de la terre»), renvoyant au protagoniste de La Route des Flandres, roman en dialogue constant avec Les Géorgiques.

22. A. B. Duncan, "Introduction", dans C. Simon, Euvres, tome II, éd. établie par A. B. Duncan, avec B. Bonhomme et D. Zemmour, "Bibliothèque de la Pléiade ", Gallimard, 2013, p. XXX.

23. "Dans Les Géorgiques, Simon reprend des éléments de son œuvre. Les allusions sont plus explicites et plus développées que dans d'autres romans et vont vers une unification de l'œuvre entière ». A. B. Duncan, "Notice ", dans C. Simon, Euvres, tome II, op. cit., p. 1514.

24. Voir, à ce propos, M. Calle-Gruber, Claude Simon. Une vie à écrire, Le Seuil, coll. "Biographie ", 2011, p. 449.

25. Voir Ph. Forest, «Le roman du je ", dans Le roman, le réel et autres essais. Allaphbed 3, Cécile Défaut, Nantes, 2007, p. 109-199. 
Situé sous la double égide du renvoi littéraire et de l'identité, et construit comme une tragédie classique en cinq $\operatorname{actes}^{26}$, ce roman raconte trois "vécus ": l'histoire de l'ancêtre de Claude Simon, Jean-Pierre Lacombe, celle de O., personnage construit à partir du "je " narrateur de Homage to Catalonia de Georges Orwell publié en 1938, comme récit de l'expérience faite par l'écrivain anglais au cours de la guerre civile espagnole entre 1936 et 1937, et celle d'un troisième personnage vu à trois époques différentes et fondatrices de sa vie: l'enfance et sa construction relationnelle au monde, l'âge adulte avec son expérience de la guerre et de la prison, apparentée à celle de Georges dans La Route des Flandres, l'âge mûr caractérisé par la recherche des traces de l'ancêtre et la pratique de l'écriture.

La traduction des Géorgiques devait donc, il me semble, mettre en relief, avant tout, la relation que, dans ce roman, l'auteur et la narration entretiennent avec la temporalité et prendre le risque de jouer une "partie d'échecs" avec le texte de départ, tout en sachant que chaque " coup " traductif risquait d'altérer et de modifier l'architecture générale et la structure d'une œuvre où tout se tient. Une partie qui a été jouée sur une " corde raide " stylistique, suspendue au-dessus d'un abîme linguistico-culturel que Claude Simon a créé dans un équilibre parfait entre cérébralité et sensorialité, entre une indéfectible rigueur formelle de fond et un écart linguistique de surface qui touche non seulement la construction phrastique $^{27}$ mais aussi tout élément lexical et connectif qui la composent, ainsi que les combinaisons, les collocations, les occurrences.

La narration du " je " se déploie dans Les Géorgiques en zigzagant à travers des temporalités diverses, juxtaposées et/ou alternées, qui se rejoignent et se fondent dans la non-temporalité du participe présent qui fige, pour Pierre Schoentjes, "l'immobile déferlement ${ }^{28}$ » du temps.

Inspiré à l'origine par la prose de Faulkner, le participe présent devient rapidement un trait majeur et original du style de Claude Simon: " [...] le recours au participe présent qui transpose la forme en -ing [...] constitue, au fond, comme une sorte d'anglicisme à l'aide duquel le français se change en une langue va-

26. Voir M. Calle-Gruber, Claude Simon. Une vie à écrire, op. cit., p. 342.

27. Pour la construction phrastique chez Simon, voir D. Zemmour, Une syntaxe du sensible. Claude Simon et l'écriture de la perception, op. cit., p. 38-56. Voir également D. Zemmour, "Les " phrase » de Claude Simon: entre thème romanesque et réflexion linguistique ", Cahiers Claude Simon, n. 3, 2007.

28. Voir P. Schoentjes, Claude Simon par correspondance. Les Géorgiques et le regard des livres, Romanica Gandesia, XXIV, Ginevra, Librairie Droz, 2007, p. 109-199, 1995. 
guement étrangère, susceptible de produire poétiquement des effets de sens qui autrement lui seraient interdits ", relève Philippe Forest ${ }^{29}$.

Dans sa «Letture di "Histoire" ${ }^{30}$ ", Guido Neri aborde explicitement la problématique interprétative du participe présent chez Claude Simon en en soulignant les caractéristiques principales, comme la mise à jour du processus narratif, sa dépendance absolue de la temporalité du contexte, mais surtout la capacité qu'il a de faire voir une action presque "freinée dans son déroulement ${ }^{31}$ ", suspendue entre motivations et effets. Une forme verbale qui est, pour lui, « tout ensemble, présent, imparfait, passé simple, aoriste et infini historique ${ }^{32}$ ". Raison pour laquelle il a préféré recourir à une variété de solutions pour traduire le participe présent simonien. En constatant l'impossibilité en italien de construire des phrases au participe présent, il fait alterner, en fait, comme il le précise dans une note, le gérondif, des formes verbales finies, des propositions dépendantes et, sur toutes, une forme qu'il définit d' "infinitif absolu ${ }^{33}$ ".

Dans le cas des Géorgiques, il me semblait que le montage simonien n'utilisait pas la suspension temporelle du participe présent pour " freiner " l'action, mais, au contraire, pour la faire " avancer" par photogrammes ${ }^{34}$. Et encore, comme autre contrainte, le traitement du participe présent devait trouver en italien une modalité expressive constante tout le long de la narration, capable de recréer le même effet d'une écriture "déréalisante " qui confond le monde et l'image du monde, à mi-chemin entre narration et description.

Pour faire ressortir au maximum, dans la traduction, cette caractéristique, il a fallu " travailler » l'italien pour l'adapter au style de Claude Simon, à partir de certains principes fondateurs qui m’ont été inspirés par les analyses critiques de Pascal Mougin ${ }^{35}$ et de David Zemmour ${ }^{36}$ sur le style simonien, ainsi que par

29. Ph. Forest, "De la phrase et d'un certain usage du participe présent propre aux romans de Claude Simon ", Cahiers Claude Simon, n. 7, op. cit., p. 235-236.

30. G. Neri, "Letture di "Histoire" ", dans Claude Simon, Storia, op. cit., p. 360-361.

31. Ibid., p. 361 : «Così nel participio presente vedremo un'azione, ma quasi frenata nel suo farsi [...]. »

32. Ibid., p. 362: "L'uso del participio presente [...] è, tutto insieme, presente, imperfetto, passato remoto, aoristo e infinito storico ".

33. Ibid.

34. Voir à ce propos mon article "L'amour est une ombre. L'érotique du regard dans Les Géorgiques de Claude Simon », in M. Costagliola d'Abele, C. Dumoulié, C. Vecce (éd.), Eros Latin, Univ. degli Studi di Napoli "L'Orientale », Napoli, Open Archive, 2014, http://opar.unior.it/1865/

35. P. Mougin, L'Effet d'image, L'Harmattan, 1997.

36. D. Zemmour, Une syntaxe du sensible. Claude Simon et l'écriture de la perception, op. cit. 
celles, très innovantes, de Carolina Diglio sur les techniques d'écriture des « Nouveaux romanciers ${ }^{37}$."

Comme le démontre Pascal Mougin dans L'Effet d'image, l'impulsion imprimée à l'action et son avancement sont donnés dans l'œuvre de Claude Simon par la juxtaposition et la succession réitérées de participes présents, qui arrivent jusqu'à occuper des pages entières, suivant une dynamique rythmique liée à la respiration et au souffle, selon David Zemmour et d'autres critiques ${ }^{38}$. Rythme que j'ai ressenti comme "ondoyant ", répliqué dans l'effet graphique du «a » et $\mathrm{du}$ " $\mathrm{n}$ ", dont les formes dessinent des vagues. C'est pour sa forme graphique et son caractère "ondoyants ", pour son voisinage aspectuel et de fonction avec le participe présent que j'ai choisi le gérondif comme forme verbale d'élection. Il soutient le rythme de la phrase, exalte l'aspect visuel et "en train de se faire " de l'action, avec un effet textuel quelque peu "étranger", semblable à celui que Forest retrouve dans la prose simonienne.

Le français a une structure phrastique très codifiée, avec laquelle Simon « joue » en travaillant sur le déplacement et l'" écart " positionnel, pour créer des effets de sens inédits et inhabituels qui retiennent l'attention du lecteur et amplifient le champ interprétatif. La plus grande liberté de construction de l'italien aurait pu amoindrir l'effet d'étrangeté signifiante produit par la syntaxe simonienne, mais celui-ci a été sauvegardé, en fait, par une reprise fidèle de la disposition des composants phrastiques envisagée par l'auteur ${ }^{39}$.

Caractérisé par une précision terminologique impressionnante, le lexique utilisé par Simon puise dans une grande variété de langages et de registres linguistiques appartenant, qui plus est, à des époques différentes, et plonge dans des champs sémantiques très variés tels que l'agriculture, l'équitation, la peinture, le cinéma, le théâtre, l'architecture, la langue juridique du XVIII ${ }^{\mathrm{e}}$ et du XIX ${ }^{\mathrm{e}}$ siècles, pour ne citer que les plus récurrents dans le roman.

Or, si la précision terminologique a pu poser quelques problèmes, la véritable difficulté s'est présentée, en réalité, lors des choix traductifs des nombreux cas de synonymie et de gradations lexicale, adjectivale et adverbiale, qui exercent un rôle

37. Voir en particulier Carolina Diglio, Lettura critica di "La Prise de Constantinople » di Jean Ricardou, Fasano (Br), Schena, 2011.

38. Voir D. Zemmour, Une syntaxe du sensible. Claude Simon et l'écriture de la perception, op. cit., p. 50.

39. Procédure suivie aussi pour ce qui concerne l'utilisation que Claude Simon fait des articles contractés dans les énumérations, ajoutés ou omis en fonction de leur présence dans le texte de départ. Plus délicat a été le choix effectué à propos des adjectifs possessifs, dont l'usage est plus abondant en français qu'en italien: pour les introduire, là où ils n'étaient pas nécessaires en italien, j'ai suivi un critère de significativité dans l'économie textuelle. 
fondamental dans la syntaxe simonienne. Fonctionnant comme un effet d'enrichissement prismatique, elles contribuent de manière déterminante, à la création d'une action comme "vision».

Cette difficulté a été de plus accrue par le fait que la ressemblance aspectuelle du lexique entre le français et l'italien ne comporte pas systématiquement une correspondance de sens.

Quelques exemples: citons la gradation adverbiale de "sans doute ", "peutêtre " " probablement ", qui puise dans le domaine de la conjecture et qui constitue un trait stylistique majeur des Géorgiques. Ce type de gradation a été rendu en italien respectivement par "probabilmente ", "forse ", " con ogni probabilità ", pour garder les valeurs de caractérisation spécifique à l'intérieur du texte. $\mathrm{Ou}$ encore la synonymie adjectivale qui sert à décrire les toiles d'araignées: "toiles scintillantes et polygonales ", "réseau étincelant et géométrique ${ }^{40}$ ", pour laquelle il a fallu respecter les contiguïtés sémantiques "brillanti/scintillanti » en les harmonisant à leurs récurrences dans l'œuvre entière.

Ou encore, la gradation de sens que l'on retrouve dans la séquence "trêve/ répit/suspension d'armes ", rendue en italien avec "tregua/pausa/sospensione " ${ }^{41}$.

Dans la traduction il m'a semblé important de reproduire fidèlement les récurrences, pour sauvegarder le système intratextuel d'occurrences et de renvois lexicaux.

Je voudrais en outre souligner deux problèmes spécifiques qui ont retenu mon attention: la traduction des toponymes et le lexique utilisé dans les parties consacrées à $\mathrm{O}$., pendant la guerre civile espagnole. J'ai traduit en italien tous les toponymes des endroits cités concernant les guerres napoléoniennes et la Seconde Guerre mondiale, en considération de leur appartenance à une mémoire collective nationale italienne. Je n'ai pas modifié dans la traduction les graphies approximatives des localités italiennes citées dans les lettres, et cela pour préserver leur aura d'authenticité ${ }^{42}$.

Plus délicate et complexe a été la traduction des parties concernant O., car ce texte inspiré d'Homage to Catalonia, est le siège d'une expérience scripturale très sophistiquée. Comme l'explique Claude Simon à Jérôme Lindon:

40. Voir C. Simon, Les Géorgiques, dans C. Simon, Euvres, tome II, op. cit., p. 677 et C. Simon, Le Georgiche, op. cit., p. 43.

41. Voir C. Simon, Les Géorgiques, dans C. Simon, Euvres, tome II, op. cit., p. 731 et C. Simon, Le Georgiche, op. cit., p. 101.

42. Choix que l'on retrouve aussi dans l'édition de la Pléiade, sortie une année plus tard: «[...] les fautes et particularités ou disparates de graphie et de typographie des écrits de LSM (ou autres documents d'époque) tels que Simon a choisi de les transcrire ont été respectés ", C. Simon, Euvres, tome II, op. cit., p. 1522. 
ce n'est pas un journaliste anglais qui " raconte " mais l'auteur/narrateur Claude Simon qui d'après un ouvrage écrit par Orwell raconte l'aventure en Espagne d'un « je " fabriqué par Orwell, qui n'est pas (ne peut pas être Orwell). O. est donc lui-même un personnage fabriqué par Claude Simon d'après le «je » fabriqué par Orwell ${ }^{43}$.

Dans l'intention de donner au lecteur italien les mêmes points de repère lexicaux et les mêmes références intertextuelles que ceux dont bénéficient les lecteurs français, j'ai fait dialoguer ma traduction avec celle d'Homage to Catalonia, réalisée par Riccardo Duranti dans la collection Meridiani Mondadori en 2000.

En conclusion, je veux rappeler que pour ma traduction, publiée en 2012, j'ai utilisé l'édition Minuit de 1981. Je regrette de ne pas avoir pu bénéficier de l'apport informatif et critique de l'édition de la Pléiade et de ses corrections typographiques ${ }^{44}$, mais je suis contente d'avoir eu, pour cela, la possibilité de transgresser les " ordres » de mon éditeur, en refusant, avec obstination, de fermer une parenthèse restée ouverte dans l'édition de 1981, car j'avais le sentiment qu'il s'agissait d'un acte volontaire et non d'une distraction éditoriale. Comme le précise la note 37 de l'édition de la Pléiade, Simon "ne fait aucun commentaire, du moins par écrit, quand Fletcher [le traducteur des Géorgiques en anglais, ndr] lui demande où fermer la parenthèse qui s'ouvre p. 702, 1. 22, après "il y a une halte" ${ }^{45}$ ".

Cette parenthèse restée ouverte, en suspens, est devenue pour moi, au même titre que le participe présent/gérondif, l'emblème du style simonien, un symbole de sa poétique, d'une écriture qui met à nu l'ouverture du monde, son insaisissabilité, sa fermeture impossible.

43. Lettre de Claude Simon à Jérôme Lindon, citée par A. Duncan dans C. Simon, Euvres, tome II, op. cit., p. 1512.

44. Voir à ce propos ibid. " Notes sur le texte ", p. 1521-1522 et ibid. note n. 37, p. 1529.

45. Ibid. 